\title{
All about Mycobacterium simiae in Brief
}

\section{Mouhamad Nasser*}

American University of Beirut- Medical Center, Lebanon

\begin{abstract}
More than hundreds pathogens of mycobacterium have been identified till now but a minority of these bugs cause diseases in humans. M. simiae, an emerging bacterium that has been discovered recently, commonly recovered from human sputum especially in patients with underlying lung diseases. Most commonly this bacterium is a bystander rather than a true culprit. Such differentiation is critical to avoid unnecessary long term treatment not free of side effects.
\end{abstract}

Keywords: Non-tuberculous Immunocompetent; Lung diseases

mycobacterium; $\quad$ M. simiae;

\section{Introduction}

Different terms are used to define non-tuberculosis mycobacteria $[\mathrm{NTM}]$ including atypical mycobarcteria and mycobacteria other than tuberculosis [MOTT]. However, NTM terminology is the most used name worldwide. NTM, an emerging entity, includes more than 100 species with variable microbiological features, clinical manifestations and significances. Nevertheless, NTM therapy also differs between species.

NTMs are rods shaped bacilli similar to Mycobacterium tuberculosis [MTB]. In 1959, Runyon classified these bacteria into four groups according to their ability to form pigment after exposure to light or dark and the rapidity of their growth as well (Table 1) [1].

The majority of human pathogens are slowly grower like M. kansasi, M. marinum, M. avium, M. intracellular, M. simiae [1]. However, M. simiae is the only niacin-positive MOTT, which makes it easily confused with Mycobacterium tuberculosis [2]. M. simiae like other NTM is a ubiquitous organism with huge environmental reservoirs, such as natural and municipal water, soil, aerosols, protozoan, animals, and humans [3].

In 1965, M. simiae was initially isolated from maccacus rhesus monkeys [4]. It was first reported in Israel, Cuba, and south western America and then reports from middle east and Asia identified the presence of this emerging specie [2,5].

\section{Epidemiology}

The incidence of diseases attributed to NTM is not well established, due to underreporting of new cases. On the other hand, the frequency of respiratory NTM is increasing worldwide. Data from Australia showed higher incidence in 2005 compared to 1999 [6,7].

\section{Clinical manifestation}

NTM have a broad spectrum of clinical manifestations. They can cause either symptomatic or asymptomatic infections. Four clinical syndromes account for nearly all cases: pulmonary diseases, lymphadenitis, skin or soft tissue diseases, and disseminated diseases especially in AIDS patients. All four clinical syndromes are increasing in frequency, particularly in immunosuppressed hosts [4]. Rarely $M$. simiae can be a cause of central line related blood stream infection especially in immunosuppressed patients [8]. M. simiae is commonly isolated from respiratory specimen without being considered as a true pathogen especially in immunocompetent patients $[9,10]$.

Pulmonary symptoms due to $M$. simiae are nonspecific. Infected patients frequently present with cough, sputum production, hemoptysis, sweating, weight loss, low grade fever, and dyspnea [4,11]. Patients with underlying lung diseases such as prior pulmonary tuberculosis or silicosis, chronic obstructive pulmonary disease [COPD], and noncystic fibrosis bronchiectasis have higher risk for $M$. simiae than healthy people [11].The association between $M$. simiae and cystic fibrosis was also been described [12]. Furthermore, other co-morbidities, such as Diabetes Mellitus, cardiovascular diseases, and malignancies could also predispose to M. simiae infection [13]. Other than that, M. simiae can cause disseminated disease in immunocompromised patients mainly HIV [14]. For long time this organism was thought to cause disseminated infections only in immunocompromised individuals. However, recent reports showed that disseminated M. simiae can also be seen in immunocompetent patients [10]. M. simiae may rarely be a cause hematogenous spread from pneumonia, leading to multifocal osteomyelitis in immunocompetent patient [15].

\section{Diagnosis}

Differentiation between true M. simiae infection and colonization is critical since treatment of infected cases is recommended for at least one year after negative culture. Thus, the American Thoracic Society (ATS) and Infectious Disease Society of America (IDSA) developed guidelines criteria to diagnose NTM pulmonary diseases (Table 2).

In addition to microbiologic information, these criteria include data about host factors, clinical, and radiological findings consistent with pulmonary diseases which mainly focused on recurrent isolation of mycobacteria from sputum or isolated from at least one bronchial wash in symptomatic patients [16]. Tuberculin skin test (TST) is not a specific test as positive results are seen in different types of mycobacterium. TST is positive in $76.9 \%$ of $M$. simiae pulmonary cases [4].

\begin{tabular}{|c|l|l|}
\hline Produce yellow pigment in light & Slowly growing & Runyon I \\
\hline Yellow pigment in light and dark & Slowly growing & Runyon II \\
\hline No pigment & Slowly growing & Runyon III \\
\hline No pigment & Rapidly growing & Runyon IV \\
\hline
\end{tabular}

Table 1: NTM Runyon Classification

*Corresponding author: Mouhamad Nasser, Pulmonary/Critical Care Medicine and American University of Beirut- Medical Center, Lebanon, Tel: 961-1-350000; E-mail: mohammad-nasser@hotmail.com

Received October 27, 2014; Accepted December 27, 2014; Published December 29, 2014

Citation: Nasser M (2014) All about Mycobacterium simiae in Brief. J Med Microb Diagn 4: 175. doi:10.4172/21610703.1000175

Copyright: (c) 2014 Nasser M. This is an open-access article distributed under the terms of the Creative Commons Attribution License, which permits unrestricted use, distribution, and reproduction in any medium, provided the original author and source are credited. 
Pulmonary symptoms + Radiographic findings that include:

-Nodular or cavitary opacities on chest radiograph.

OR

- Multifocal bronchiectasis with multiple small nodules on HRCT chest. AND

Clinical and Radiographic features

Appropriate exclusion of other diagnoses.

-Positive results of culture from at least two separate sputum specimen.

OR

-Positive result of culture from at least one bronchial wash or lavage.

- Mycobacterial histopathologic features (granulomatous inflammation or AFB) from transbronchial or other lung biopsy and positive culture for NTM.

OR

-Mycobacterial histopathologic features (granulomatous inflammation or AFB) on Biopsy and one or more positive sputum or bronchial washings for NTM.

Abbreviations: ATS: American Thoracic Society; ERS: European Respiratory Society; HRCT: High Resolution Computed Tomography; AFB: Acid Fast Bacilli. Table 2: ATS/ERS criteria for pulmonary NTM diagnosis

\section{Radiographic findings}

Radiographic findings with NTM pulmonary infections could be very similar to those seen in MTB findings. In addition, most patients with NTM have structural lung diseases. Typical radiographic features include nodular lesions (100\%), cavitary lesions $(88.5 \%)$, and bronchiectasis $(84.6 \%)$. The middle and lower lobes are commonly involved in M. simiae infections [4].

\section{Treatment}

Although NTM might share similar clinical and radiological findings, treatment approach is different between species. Therefore identification of mycobacterium species is extremely important whenever NTM is recovered. The choice of antibiotics depends on the susceptibility pattern obtained from in vitro susceptibility tests that includes Clinical and Laboratory Standards Institute (CLSI) E-test, agarbased testing methods, and the disk elution and diffusion method [17].

M. simiae isolates are susceptible to fluoroquinolones and amikacin but resistant to all others including isoniazid, capreomycin, minocycline, doxycycline, p-aminosalicylic acid, and ethionamide [17]. However, some isolates are susceptible in vitro to trimethoprimesulfamtahoxazole and linezolid [2].

To date, there is no standard treatment yet for M. simiae, but the following drugs: clarithromycin, quinolones, ethambutol, cycloserine, and ethionamide seem to be effective. Surprisingly M. simiae isolates remain sensitive to moxifloxacin even if the isolates are resistant to ciprofloxacin [2].

Regimen including moxifloxacin, clarithromycin and a third drug to which the isolate is susceptible (like trimethoprime-sulfamtahoxazole, clofazimine, streptomycin, amikacin) is the best treatment approach [2].

Aminoglycosides and clofazimine are promising therapeutic options [18].

\section{Conclusion}

M. simiae infection is uncommon and can affect immunosuppressed and immunocompetent patients as well. Its presence in respiratory specimen could represent either true infection or colonization. The clinical manifestations and radiological findings usually resemble another infectious NTM. However early diagnosis is necessary in order to implement appropriate treatment despite limited choices in such cases.

\section{Reference}

1. Runyon Eh (1959) Anonymous mycobacteria in pulmonary disease. Med Clin North Am 43: 273-290.

2. Maoz C, Shitrit D, Samra Z, Peled N, Kaufman L, et al. (2008) Pulmonary Mycobacterium simiae infection: comparison with pulmonary tuberculosis. Eur $\mathrm{J}$ Clin Microbiol Infect Dis 27: 945-950.

3. Makovcova J, Slany M, Babak V, Slana I, Kralik P (2014) The water environment as a source of potentially pathogenic mycobacteria. J Water Health 12: 254-263.
4. Baghaei $P$, Tabarsi $P$, Farnia P, Marjani M, Sheikholeslami FM, et al. (2012) Pulmonary disease caused by Mycobacterium simiae in Iran's national referral center for tuberculosis. J Infect Dev Ctries 6: 23-28.

5. Hashemi-Shahraki A, Darban-Sarokhalil D, Heidarieh P, Feizabadi MM Deshmir-Salameh S, et al. (2013) Mycobacterium simiae: a possible emerging pathogen in Iran. Jpn J Infect Dis 66: 475-479.

6. Thomson RM (2010) on behalf of the NTM working group at the Queensland TB Control Centre and Queensland Mycobacterial Reference Laboratory Changing Epidemiology of Pulmonary Nontuberculous Mycobacteria Infections. Emerging Infectious Diseases .www.cdc.gov/eid . Vol. 16, No. 10.

7. Good RC, Snider De (1982) Isolation of non-tuberculosis mycobacterium in the United States. J.infect Dis: $146-829$

8. El Helou G, Hachem R, Viola GM, El Zakhem A, Chaftari AM, et al. (2013) Management of rapidly growing mycobacterial bacteremia in cancer patients. Clin Infect Dis 56: 843-846.

9. Hashemi-Shahraki A, Darban-Sarokhalil D, Heidarieh P, Feizabadi MM Deshmir-Salameh S, et al. (2013) Mycobacterium simiae: a possible emerging pathogen in Iran. Jpn J Infect Dis 66: 475-479.

10. Balkis MM, Kattar MM, Araj GF, Kanj SS (2009) Fatal disseminated Mycobacterium simiae infection in a non-HIV patient. Int J Infect Dis 13: e286-287.

11. Barrera L, Palmero D, Paul R, López B; Grupo de Investigacion de M simiae. (2010) [Disease due to Mycobacterium simiae and "Mycobacterium sherrisii" in Argentina]. Medicina (B Aires) 70: 343-346.

12. Levy I, Grisaru-Soen G, Lerner-Geva L, Kerem E, Blau H, et al. (2008) Multicenter cross-sectional study of nontuberculous mycobacterial infections among cystic fibrosis patients, Israel. Emerg Infect Dis 14: 378-384

13. Shitrit D, Peled N, Bishara J, Priess R, Pitlik S, et al. (2008) Clinical and radiological features of Mycobacterium kansasii infection and Mycobacterium simiae infection. Respir Med 102: 1598-1603.

14. Al-Abdely HM, Revankar SG, Graybill JR (2000) Disseminated Mycobacterium simiae infection in patients with AIDS. J Infect 41: 143-147.

15. Chung KP, Tsai TH, Huang YT, Hsueh PR (2009) Refractory bacteremia and osteomyelitis resulting in fatal bacteremic pneumonia with multiorgan failure caused by Mycobacterium simiae in a non-human immunodeficiency VirusInfected Adult. J Clin Micro biol. 47: 3777-3779.

16. Griffith DE, Aksamit T, Brown-Elliott BA, Catanzaro A, Daley C, et al. (2007) An official ATS/IDSA statement: diagnosis, treatment, and prevention of nontuberculous mycobacterial diseases. Am J Respir Crit Care Med 175: 367-416.

17. Li G, Lian LL, Wan L, Zhang J, Zhao X, et al. (2013) Antimicrobial susceptibility of standard strains of nontuberculous mycobacteria by microplate Alamar Blue assay. PLoS One 8: e84065.

18. Valero G, Peters J, Jorgensen JH, Graybill JR (1995) Clinical isolates of Mycobacterium simiae in San Antonio, Texas. An 11-yr review. Am J Respir Crit Care Med 152: 1555-1557. 